\title{
Risk Factors for the Development of Incisional Hernia Following Roux-en- Y Gastric Bypass Surgery
}

\author{
Michael I. Horowitz and I. Michael Leitman ${ }^{*}$, \\ ${ }^{a}$ Department of Surgery, SUNY Downstate Medical Center, Brooklyn, NY 11203, USA \\ ${ }^{b}$ Department of Surgery, Beth Israel Medical Center, New York, NY 10003, USA
}

\begin{abstract}
Roux-en-y gastric bypass (RYGB) has become the most common operation in the United States for morbid obesity. Even in the laparoscopic era many procedures are still done via laparotomy. Incisional hernias are a complication of any abdominal surgery with obese patients being at increased risk. Patients undergoing open RYGB over 6 years were reviewed to evaluate the risk factors for the development of incisional hernia. 444 adults (88 male, 3566 female) using ASBS and NIH criteria who underwent open RYGB were studied. Patients with asthma and a history of steroid use were all off steroids for at least 6 months prior to surgery. Lesser curvature RYGB was performed through a 10-14 cm upper abdominal incision. The linea alba was closed using running 1-0 polydiaxone sulfate (PDS) sutures. The mean follow up was 2 years. The incidence of incisional hernia was $18.7 \%$, based upon post-operative office follow-up and/or CT scan. Overall, $130(29.3 \%)$ required post-operative local wound care for drainage, $44(33.8 \%)$ of which developed incisional hernias. $93(23.3 \%)$ had a past medical history that included asthma, $32(34.4 \%)$ of which developed incisional hernias. There was a statistically significant association between a history of asthma and post-operative wound infection with the development of incisional hernia. In this study, other variables, such as gender, prior incisional hernia repair, prior abdominal surgery, pre-operative BMI, history of smoking, diabetes, $\% \mathrm{EWL}$ and post-operative pneumonia were not found to be associated with incisional hernia. Measures to minimize post-operative wound infection or excessive coughing in patients with asthma may reduce the incidence of incisional hernia following RYGB.
\end{abstract}

\section{INTRODUCTION}

Obesity (BMI $>40 \mathrm{~kg} / \mathrm{m}^{2}$ ) is a chronic disease that affects roughly 31 percent of the US population, with 5 percent of adults in the United States being morbidly obese [1, 2]. It is associated with significant morbidity and mortality, and is the cause of death in 280,000 to 325,000 Americans annually [3]. Gastric bypass surgery offers resolution or significant improvement of many of the co-morbid conditions associated with obesity. It has thus far proven to be an effective long-term solution for weight loss in the morbidly obese population, with demonstrable long-term continued weight loss and resolution of many co-morbid illnesses [4]. Although there has been a trend toward laparoscopic surgery, many open gastric bypass operations are being performed annually, usually through an upper midline incision. Obesity clearly increases the risk of incisional hernia [8-11] but other risk factors may predispose patients for the development if incisional hernia following gastric bypass surgery. Respiratory problems and wound infection have been associated with an increased risk for the development if incisional hernias following all general surgical operations $[6,11]$.

The present study investigates the risk factors for the development of incisional hernia occurrence in patients who underwent open RYGB.

\section{PATIENTS AND METHODS}

Patients undergoing RYGB at a single institution over a 4 year period, between April 1999 and June 2005, were

*Address correspondence to this author at the Department of Surgery, Beth Israel Medical Center, New York, NY 10003, USA;

E-mail: MLeitman@chpnet.org reviewed to evaluate the risk factors for incisional hernia after open RYGB. 444 adults (88 male, 356 female) using American Society for Bariatric Surgery and National Institutes of Health (NIH) criteria who underwent open RYGB were studied. Patients with asthma and a history of steroid use were all off steroids for at least 6 months prior to surgery. Steroid replacement therapy was not given perioperatively. A single dose of a first generation cephalosporin antibiotic was administered within 1 hour of the start of the operation, except in penicillin allergic patients-who received one dose of clindamycin and gentamicin. Antibiotics were not prescribed following surgery. Lesser curvature RYGB was performed through a 10-14 cm upper abdominal incision by a single surgeon (IML). In all patients the linea alba was closed using running 1-0 polydiaxone sulfate (PDS) sutures. No mesh was utilized in any patients for abdominal fascial closure. Saline lavage of the fascial closure was used utilized to decrease wound contamination. The skin was then reapproximated with surgical clips that were removed 12 days after surgery. The mean follow up for this group was 2 years. 25 patients were lost to follow-up and were not included in this analysis. Patients had routine follow-up examinations and wound care. Wound collections that were not purulent were treated with simple drainage, in the absence of fever, cellulitis or elevated white blood cell count. Patients with documented wound infections were treated with oral antibiotics, based upon cultured organisms and antibiotic sensitivity reports.

CT scans were generally performed as needed for the evaluation of gastro-intestinal symptoms or abdominal pain following surgery. 
Data were subjected to univariate and multivariate analysis. A p-value of less than 0.05 was considered statistically significant.

\section{RESULTS}

444 patients (88 male, 356 female) were followed for a mean of 25 months (11-66). The average age was 43.1 years (21-64). Percentage of excess weight lost averaged 44.2\% (3-98). The average length of hospital stay was 4.6 days (344). Six patients (1.3\%) developed anastomotic leak during the study period. None of the patients required re-operation during the same admission for fascial dehiscence. The thirtyday morality was $0.7 \%$. Two deaths were due to septic complications; the other resulted from post-operative cerebrovascular accident.

The incidence of incisional hernia during the study period was $18.7 \%$ (83 patients), based upon post-operative office follow-up physical examination and/or CT scan. Overall, 130 patients $(29.3 \%)$ required post-operative local wound care for drainage, 44 (33.8\%) of which developed incisional hernias $(p=0.007)$. In the entire study group, 93 patients (20.9\%) had a past medical history of asthma requiring medical therapy of which 32 patients (34.4\%) developed incisional hernias $(p=0.005)$. There was an association between a history of asthma and post-operative wound infection with the development of incisional hernia following open RYGB. Other variables, such as age, gender, prior incisional hernia repair, prior abdominal surgery, pre-operative $\mathrm{BMI}, \% \mathrm{EWL}$, history of smoking, diabetes, and postoperative pneumonia were evaluated independently and were found not to be associated with significant increases in the development of incisional hernias after RYGB.

\section{DISCUSSION}

The general incidence of incisional hernia following abdominal surgery through the midline is reported to be 4 $19 \%$ [17] and similar rates have been reported following gastric bypass surgery [18-21]. Many factors have been linked to the development of hernia following laparotomy such as male gender, advanced age, poor nutrition, diabetes, renal failure, steroid use, malignancy, anemia, and of course, obesity [18]. In this study several factors were evaluated independently to ascertain whether any of them increased the likelihood of development of an incisional hernia postoperatively. Factors evaluated were gender, prior incisional hernia repair, prior abdominal surgery, pre-operative BMI, history of smoking, diabetes, and post-operative pneumonia, as well as asthma and the necessity for post-operative local wound care. Post-operative wound infection rates following open gastric bypass surgery are high. Christou and others [19] reported a wound infection rate of $20 \%$, more than 4 times higher than expected for similar clean-contaminated cases, which they correlated to the development of hernia. The development of an incisional hernia following postoperative wound infection has been reported by others, and may even be the most important risk factor for the development of incisional hernia $[6,11,15]$. This may be the result of impaired collagen synthesis at infected wound sites.

Obesity appears to cause a greater risk of incisional hernia than concomitant steroid use [19]. The present study also demonstrated that patients with preexisting asthma were found to be more likely to develop incisional hernias postoperatively. Asthma has been noted to place patients at increased risk for hernia development by others [22]. This is likely due to the increased intra-abdominal pressure caused by excessive coughing. As reported by Sugarman [16], increased intra-abdominal pressure may play a significant role for incisional hernia following gastric bypass surgery. It is clear that laparoscopic gastric bypass surgery has reduced the incidence of incisional hernia [23].

One notable finding in this study was that there was no statistically significant correlation between prior incisional hernia and subsequent recurrence of incisional hernia. Many studies have clearly shown that there is indeed a significant correlation to this effect. It is possible that no correlation was found in this study due to a type 2 statistical error i.e. that the sample size was too small to fully evaluate this relationship. Had the sample size been larger it is probable that this relationship would have correlated with previously published reports.

For this retrospective review, patients were very carefully screened and selected. Those that were lost to follow-up or fell outside the scope of what is discussed here were excluded from the study. All data is based on a sample of patients that had the same operation, for the same illness. The variables studied were screened for in all of the patients in the study and available in the patients' charts for review.

\section{CONCLUSIONS}

The development of incisional hernia following gastric bypass surgery is common. Measures to minimize postoperative wound infection or excessive coughing and straining in patients with asthma may reduce the incidence. The incidence may be decreasing with the use of synthetic mesh placement and as the percentage of laparoscopic gastric bypass operations increases. However, this still remains an important cause of morbidity after bariatric surgery.

\section{REFERENCES}

[1] Flegal KM, Carroll MD, Ogden CL, Johnson CL. Prevalence and trends in obesity among US adults, 1999-2000. JAMA 2002; 288: 1723.

[2] Buchwald H, Avidor Y, Braunwald E, et al. Bariatric surgery: a systematic review and meta-analysis, JAMA 2004; 292(14): 172437.

[3] Allison, DB, Fontaine, KR, Manson, JE, Stevens J, Vanltallie TB. Annual deaths attributable to obesity in the United States. JAMA 1999; 282: 1530-8.

[4] White S, Brooks E, Jurikova L, Strubbs RS. Long-term outcomes after gastric bypass. Obes Surg 2005; 15(2): 155-63.

[5] Must A, Spadano J, Coakley EH, et al. The disease burden associated with overweight and obesity. JAMA 1999; 282: 1523-9.

[6] Chrstou NV, Jarand J, Sylvestre JL, McLean AP. Analysis of the incidence and risk factors for wound infections in open bariatric surgery. Obes Surg 2004; 14(1): 16-22.

[7] Sugerman HJ, Starkey JV, Birkenhauer R. A randomized prospective trial of gastric bypass versus vertical banded gastroplasty for morbid obesity and their effects on sweets versus non-sweets eaters. Ann Surg 1987; 205: 613-24.

[8] Bonatti H, Hoeller E, Kirchmayr W, Muhlmann G, Zitt M, Aigner $\mathrm{F}$, Weiss $\mathrm{H}$, Klaus A. Ventral henia repair in bariatric surgery. Obes Surg 2004; 14(5): 655-8.

[9] Regnard JF, Hay JM, Rea S, Fingerhut A, Flamant Y, Maillard JM. Ventral incisional hernias: incidence, date of recurrence, localization and risk factors. Ital J Surg Sci 1998; 18(3): 259-65.

[10] Sauerland S, Kornkov M, Kleinen T, Arndt M, Paul A. Obesity is a risk factor for recurrence after incisional hernia repair. Hernia 2004; 8(1): 42-6. 
[11] Yahchouchy-Chouillard E, Aura T, Picone O, Etienne JC, Fingerhut A. Incisional hernias. I. Related risk factors. Dig Surg 2003; 20(1): 3-9.

[12] Mun EC, Blackburn GL, Matthews JB. Current status of medical and surgical therapy for obesity, Gastroenterology 2001; 120(3): 669-81.

[13] www.oit.edu/zimmermg/ pathology/hernia.htm

[14] Brolin RE. Prospective randomized evaluation of midline fascial closure in gastric bariatric operations. American Journal of Surgery 1996; 172: 328-31.

[15] Chang G, Chan CK. A review of incisional hernia repairs: postoperative weight loss and selective use of the mesh repair. Hernia 2005; 9(1): 37-41.

[16] Sugerman HJ. Effects of increased intra-abdominal pressure in severe obesity. Surg Clin North Am 2001; 81(5): 1063-75.

[17] Hoer J, Lawong G, Klinge U and Schumpelick. Factors influencing the development of incisional hernia. Chirurg 2002; 73 (5): 474480.

[18] Sorensen LT, Hemmingsen UB, Kirkeby LT, Kallehave F and Jorgenson LN, Smoking is a risk factor for incisional hernia, Arch Surg 2005; 140 (2): 119-123.
[19] Sugerman HJ, Kellum JM, Reines HD, DeMaria EJ, Newsome HH, Lowry JW. Greater risk of incisional hernia with morbidly obese than steroid-dependent patients and low recurrence with prefascial polypropylene mesh. Am J. Surg 1996; 171 (1): 80-84.

[20] Nguyen NT, Goldman C, Rosenquist CJ, Cole CJ, Lee SJ and Wolfe BM. Laparoscopic versus open gastric bypass: a randomized study of outcomes, quality of life, and costs. Ann Surg 2001; 234 (3): 279-289.

[21] Strzelczyk J, Czupryniak, Loba J, Wasiak. The use of polypropylene mesh in the midline incision closure following gastric by-pass reduces the risk of postoperative hernia, Langenbecks Arch Surg 2002; 387: 7-8.

[22] Veillette G, MacGillivray D, Whalen G. Practical experience with the Stoppa repair of ventral/incisional hernias. Conn Med 2001; 65(2): 67-70.

[23] Podnos YD, Jiminez JC, Wilson SE, Stevens CM, Nguyen NT. Complications after laparoscopic gastric bypass: a review of 3464 cases, Arch Surg 2003; 138(9): 957-961. 